\title{
NAO and SHAO participation in the near-Earth space observations
}

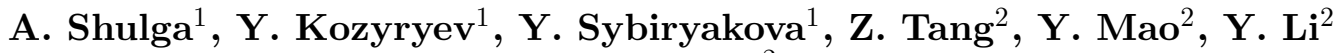 \\ and $\mathrm{Y} . \mathrm{Yu}^{2}$ \\ ${ }^{1}$ Nikolaev Astronomical Observatory \\ Nikolaev, 54030, Ukraine \\ email: avshulga@mail.ru \\ ${ }^{2}$ Shanghai Astronomical Observatory \\ Shanghai, 200030, China \\ email: zhtang@shao.as.cn
}

\begin{abstract}
The joint observations of space debris are conducted by NAO and SHAO. The main task of observations is precise estimation of the hazard collisions of SD with operating satellites. Observations of the near earth asteroids (NEA) on the distance of less than $0.05 \mathrm{AU}$, according to the NEODyS program, are conducted in NAO. The main task is to obtain the large number of precise observations of the NEAs during their closest approach to the Earth.
\end{abstract}

Keywords. space debris, near earth asteroids

\section{Telescopes and method of observation}

The observations are carried out by combined CCD observation method (developed by NAO in 2000). The combined CCD observation method is based on using of CCD time delay and integration mode (TDI) and obtaining images of object and reference stars in different frames. To obtain images of space debris or fast NEAs using TDI mode, the CCD matrix columns must be parallel to the object motion direction. To rotate the CCD camera around the optical axis of the lens a special mechanical device was developed in NAO (Shulga et al. 2008). Exposure time of the classical TDI imaging is equal to the time of object passage through the field of view, and is inefficient for space debris and NEAs observation. In most cases this fixed exposure is too long, and may cause the oversaturation of frame background and object image or distortion of the object image due to acceleration of apparent motion of the space debris or NEAs. Therefore, a special "short" TDI imaging technique was developed in NAO (Shulga et al. 2011).

Three telescopes in NAO and one telescope in SHAO were designed and used for observation of space debris and NEAs. The main parameters of telescopes are given in Table 1. All telescopes are equipped with U9000 Apogee CCD cameras, provided by SHAO.

Table 1. The main telescope parameters.

\begin{tabular}{|l|c|c|c|}
\hline Name of telescope & Observatory & Diameter[mm] & Focal length [mm] \\
\hline Fast robotic telescope (FRT) & NAO & 300 & 1500 \\
\hline Mobitel-KT50 & NAO & 500 & 3000 \\
\hline Satellite tracking camera AFU-75 & NAO & 267 & $\mid$ \\
\hline Large-field of view telescope RDS-30 & SHAO & 300 & 750 \\
\hline
\end{tabular}




\section{Results}

Regular joint observations of low Earth space debris have been carried out in NAO and SHAO since 2008. The orbital elements and observation errors are calculated using software developed by NAO and SHAO. The following standard deviation for both coordinates: (1-3)" for the AFU-75, (2-5)" for the RDS-30, were obtained during calculations of the orbital elements.

During 2008-2012, 1702 positions of 109 NEAs were obtained in NAO (observatory code 089), and 30 of them were of potentially hazardous asteroids. The positions were sent to the IAU Minor Planet Center. The 178 positions of 5 NEAs with magnitude of (12.815.9), apparent motion of (4.6-68.3)" / min and at distance of (0.01-0.5) AU were obtained with the FRT. The standard deviations of position of 5 NEAs for both coordinates are (0.23-0.43)". The 1524 positions of 104 NEAs with magnitude of (9.5-18.5), apparent motion of (0.5-306.3) "/ min and at distance of (0.009-0.98) AU were obtained with the Mobitel-KT50. The standard deviations of positions for both coordinates are (0.23-0.47)".

\section{References}

Shulga, O., Kozyryev, Y., \& Sybiryakova, Y. 2008, Proc. of IAU Symposium 248, pp. 128-129.

Shulga, O., Kozyryev, Y., \& Sybiryakova, Y. 2011, Proc. Of Gaia follow-up network for solar system objects workshop, pp. 97-100. 